\title{
The Automatic Sampler for UV Spectroscopy Toxicity Detection: Based on the Perspective of Binding Information with the Carrier Protein
}

\author{
Xiangshuai Li, Xiangfen Li, Zishi Wang*, Hongliang Xu* \\ Engineering Research Center of Pesticide of Heilongjiang Province, College of Advanced Agriculture \\ and Ecological Environment, Heilongjiang University, Harbin 150080, China \\ E-mail address: xuhongliang@hlju.edu.cn (H.Xu); 2016048@hlju.edu.cn (Z.Wang) \\ *Corresponding Author
}

\begin{abstract}
Studies have shown that high toxicity at all stages of drug development is a major limiting factor in getting a new drug to market. At present, a number of toxicity assay methods have been published in the literature. Most of them rely mainly on the UV spectrometer and manual injection. However, the manual injection has low accuracy of detection results, long detection time, complicated operation, cross-contamination, and inadequate safety factor. Therefore, it is necessary to develop an automatic sampler that is used in combination with a platform for predicting drug toxicity based on carrier protein binding information and an ultraviolet spectrophotometer. The automatic sampler for $U V$ spectroscopy toxicity detection uses the quartz cell gripper to clamp the quartz cell and realizes the precise automatic sample injection function through the position sensor. Through the movement simulation, the whole moving process is smooth and without interference, and the movement position of the quartz cell is accurate. The research and development of the automatic sampler for UV spectroscopy toxicity detection will significantly promote the precise detection of toxicity in the early stage of drug development. The development of UV spectral toxicity detection autosampler will provide hardware support for the precise detection of toxicity in the early stage of drug development. At the same time, it will significantly shorten the drug $R \& D$ process and save a lot of $R \& D$ expenses for drug $R \& D$.
\end{abstract}

Keywords: Ultraviolet Spectroscopy. Quartz cell gripper. Toxicity detector. Position Sensor.

\section{Introduction}

Ultraviolet-visible spectroscopy is a powerful analytical method in modern laboratories, which has advantages of many kinds of detection, wide range of measurable concentrations, good reproducibility, simple operation and low analysis cost[1]. It is widely used in drug research and development, agriculture, bioengineering, chemical and chemical industries. Drug toxicity is one of the critical factors that affect the success of drug development. UV-Vis spectrophotometers are often used to detect the toxic combination of drugs and carrier proteins, binding information such as the fluorescence quenching mechanism, binding distance and other information can be obtained.

Problems such as manual sampling, drug residues, difficult cleaning, low safety factor and large errors have become the main factors for the low accuracy of traditional ultraviolet-visible spectrophotometer measurement results[2]. In the process of detecting the toxic combination of drugs and carrier proteins, Quartz cells contain drugs of different toxicity, which requires the high cleanliness of quartz cells to solve the problem of large manual injection errors, long detection time, complex operation and other issues. Therefore, based on the original UV sampling device, we improved the sampling device and developed the automatic sampler for UV spectroscopy toxicity detection, which is a combination of "carrier protein binding information-toxicity relationship(CPBITR)[3]" and ultraviolet-visible spectrophotometer to predict drug toxicity. The ultraviolet automatic sampling instrument is an ultraviolet-visible spectrophotometer sampling device (The device has applied for a utility model patent, Patent No. 202110178985.7.) that automatically sample samples and detects drug toxicity, which is mainly composed of Sterilization $4^{\circ} \mathrm{C}$ thermostat, temperature regulator, Constant temperature water bath tube, drug solution to be tested Storage box and USB jack, and each component is an independent part, which is conducive to changing samples, maintaining low aseptic temperature in the storage box and automatically adding samples. The buffer and protein solution is stored in a storage 
box and placed in a sterilization $4^{\circ} \mathrm{C}$ thermostat to avoid protein deformation and solution contamination. The quartz cells are automatically sampled and cleaned by the clamping device, which avoids experimental errors caused by improper operation. The USB jack is provided, which is conducive to the realization of the online spectral signal collection of the UV autosampler. The use of the "carrier protein binding information-toxicity relationship(CPBITR)" is used to automatically control the various processes of the UV sampler, which can effectively improve the safety factor, detection time and reduce errors.

The purpose of this work is to develop an automatic sampler for UV spectroscopy toxicity detection to automatically samples. Collect the ultraviolet spectrum signal by connecting the "carrier protein binding information-toxicity relationship" and the UV spectrometer. And then propose the device to reduce test errors caused by automatically control the drug to be tested liquid quantitative addition, dishware cleaning, human operation, temperature changes, quartz cells drug residues and experimental background conditions interference to shorten the detection time and improve accuracy. And finally, combined with the "carrier protein binding information-toxicity relationship," the goal of early estimation of the toxicity of drugs and carrier proteins is achieved, and lays the foundation for the early estimation of drug toxicity in the drug development process.

\section{Sampling Instrument Structure}

As shown in figure 1, the entire autosampler includes Autosampler housing (5), the sterilization $18^{\circ} \mathrm{C}$ thermostat (17) and the sterilization $4^{\circ} \mathrm{C}$ thermostat (24). The sterilization $18^{\circ} \mathrm{C}$ thermostat (17) and the sterilization $4^{\circ} \mathrm{C}$ thermostat $(24)$ are installed on both sides of the inner wall of the autosampler housing (5) respectively. The compound to be tested is mixed and filled with an organic solvent in a certain proportion into the inside of the drug solution to be tested storage box (16) be tested.

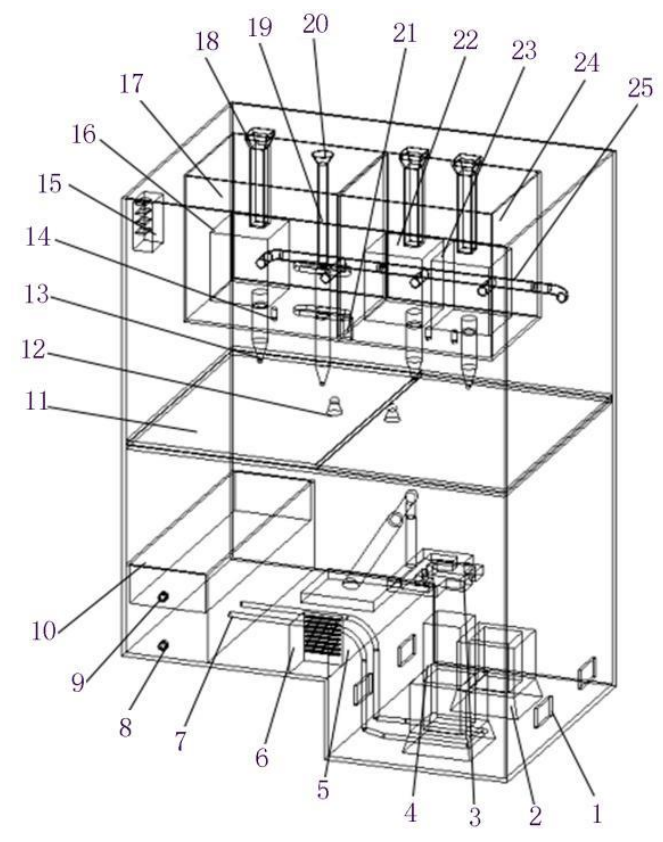

Figure 1: Assembly drawing of ultraviolet automatic sampling instrument for drug toxicity detection based on the information angle of binding with the carrier protein. (Light passing hole, 2.Quartz cell base, 3.Quartz cell gripper, 4.Quartz cell, 5.Autosampler housing, 6.temperature regulator, 7.Constant temperature water bath tube, 8.Ultrasonic water bath drainage port, 9.Ultrasonic water bath filling port, 10.Ultrasonic water bath, 11.Insulation baffle, 12.Obstacle sensing switch, 13.Injection gun head,

14.Position Sensor, 15.USB jack, 16.Drug solution to be tested storage box, 17. Sterilization $18^{\circ} \mathrm{C}$

thermostat, 18. Filling cleaning port, 19.Mixing gun, 20.Mixing gun cleaning port, 21.Temperature

Sensor, 22.Carrier protein storage box, 23.Buffer storage box, 24.Sterilization $4^{\circ} \mathrm{C}$ thermostat,

$$
\text { 25.Clean drain.) }
$$

The carrier protein storage box (22) and the buffer storage box (23) are respectively fixedly connected to the inner sides of the sterilization $4{ }^{\circ} \mathrm{C}$ thermostat (24). The prepared PBS buffer or HEPES buffer can be filled in the buffer storage box (23). The position sensor (14) is provided on the rear side of the injection gun head (13). The clean drain (25) is installed inside the sterilization $18^{\circ} \mathrm{C}$ 
thermostat (17) and the sterilization $4^{\circ} \mathrm{C}$ thermostat (24). As shown in Figure 2, the left end of the autosampler housing (5) is equipped with the USB jack (15) for data connection between the corresponding device and the external "platform for predicting drug toxicity based on the information angle of binding with carrier protein." (The platform has registered the computer software copyright in the China Copyright Protection Center, the registration number is 2021SR0226101)

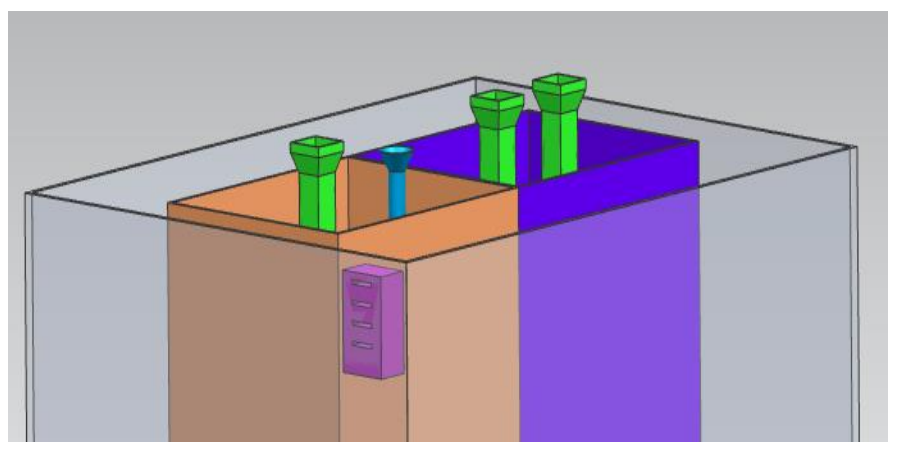

Figure 2: USB jack parts diagram.

As shown in figure 3, both sides of the front end of the ultrasonic water bath (10) are respectively processed with the ultrasonic water bath filling port (9) and the ultrasonic water bath drainage port (8). The ultrasonic water bath filling port (9) and the ultrasonic water bath drainage port (8) are used for injecting distilled water and draining the cleaning water, respectively. The constant temperature water bath tube (7) passes through the bottom of the quartz cell base (2) to provide a test condition of the corresponding temperature for the liquid to be tested in the quartz cell (4). The quartz cell (4) is placed inside the quartz cell base (2). As shown in Figure 4, the light passing hole (1) is machined on the inner wall of the autosampler housing (5) and the light passing hole (1) can correspond to the spectrum scanning hole of the UV-1780 UV-Vis Spectrophotometer.

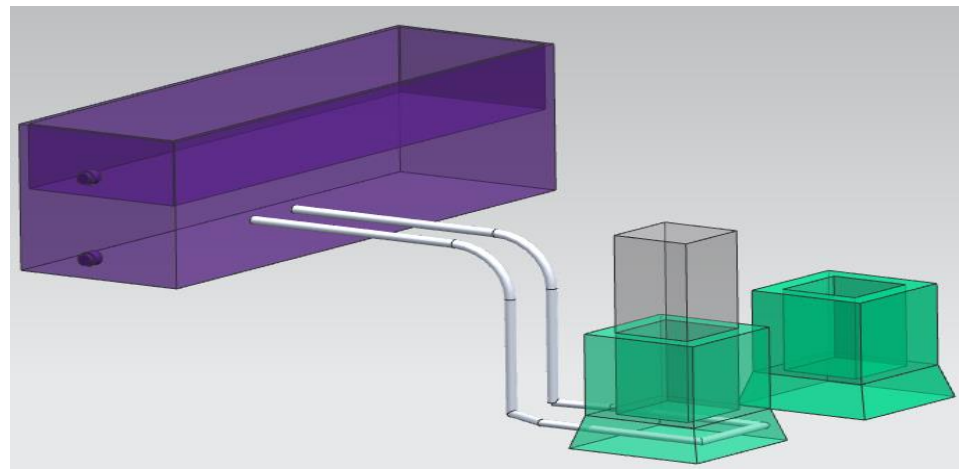

Figure 3: Constant temperature water bath pipe piping diagram.

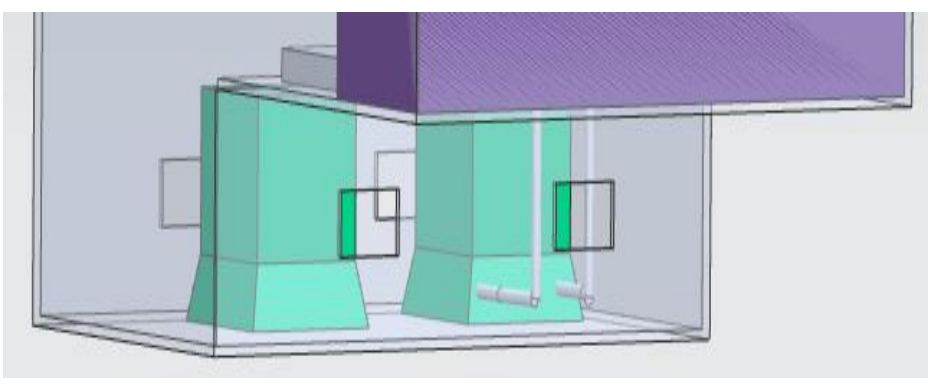

Figure 4: Light passing hole partial view.

The sterilization $4^{\circ} \mathrm{C}$ thermostat (13) and the constant temperature water bath tube (7) are equipped with the temperature sensor (21) that transmit their internal temperatures to the "platform for predicting drug toxicity based on the information angle of binding with carrier protein." It can not only monitor the storage temperature of its internal substances in real-time, but also accurately determine whether the temperature of the water bath required by the experiment is reached, so as to accurately determine the water bath time. The temperature regulator (6) has two modes of space temperature regulation and partial drying. The spatial temperature adjustment mode of the temperature regulator (6) can 
continuously and automatically adjust the temperature in the autosampler housing (5) to remain unchanged according to changes in the external temperature. As shown in figure 5, the thermal insulation baffle (11) acts as a barrier to the upper and lower temperatures inside the autosampler housing (5) to ensure that the stored solution in the upper part of the autosampler housing (5) does not deteriorate due to the high temperatures during testing in the lower part. The insulation baffle (11) is slidably connected with the inner wall of the autosampler housing (5). The obstacle sensing switch (12) is installed at the center of the inner side of the insulation baffle (11).

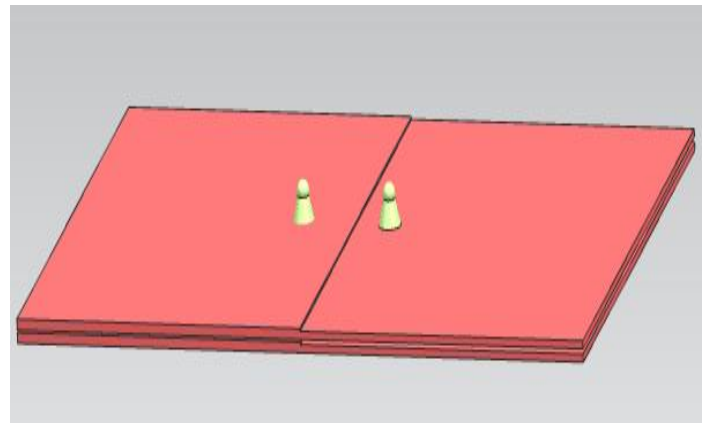

Figure 5: Insulation baffle parts diagram.

\section{The Function of the Ultraviolet Automatic Sampling Instrument}

\subsection{Automatic Cleaning Function}

Each time before using the entire device for toxicity testing, or after use. The "platform for predicting drug toxicity based on the information angle of binding with carrier protein" will automatically activate the cleaning function.

\subsubsection{Automatically Starts Cleaning Function 1}

As shown in figure 6, whenever the automatic cleaning function is turned on 1 , the connection port of the storage box and the injection gun head will be automatically closed. Pour appropriate distilled water from the filling cleaning port and the mixing gun cleaning port to the carrier protein storage box, the buffer storage box, the drug solution to be tested storage box and the mixing gun is cleaned, and the waste liquid is discharged from the drain to the outside of the device. Repeat three times. Start the drying function of the sterilization $4^{\circ} \mathrm{C}$ thermostat and the sterilization $18^{\circ} \mathrm{C}$ thermostat, and set the carrier protein storage box, buffer storage box and drug solution to be tested storage box, the mixing gun and the clean drain pipe are completely dried.

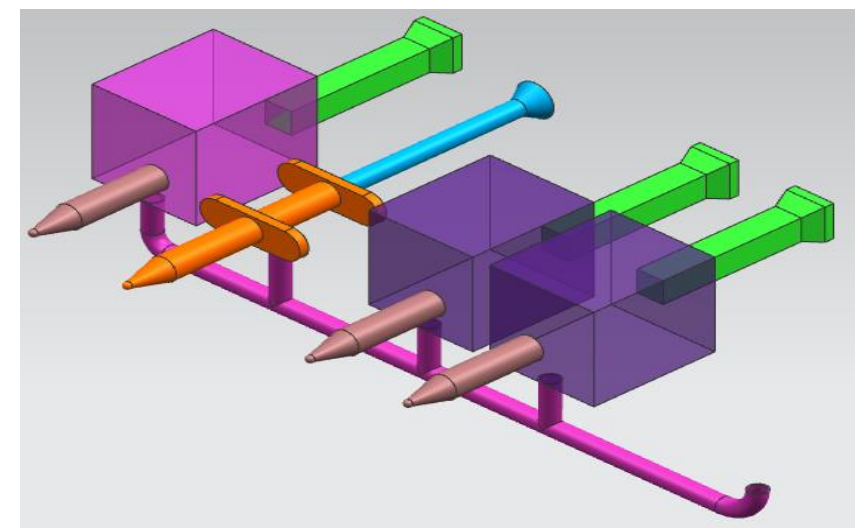

Figure 6: Cleaning system piping diagram.

\subsubsection{Automatically Starts Cleaning Function 2}

Whenever the automatic cleaning function is turned on 2, the quartz cell gripper clamps the quartz cell and puts it in the ultrasonic water bath. The ultrasonic water bath starts the ultrasonic function for 10 minutes to clean the quartz cell. The cleaning waste liquid is discharged from the ultrasonic water bath drainage port. Inject new distilled water back and forth into the ultrasonic water bath, and repeat the cleaning of the quartz cell three times. After three repeated cleanings, the quartz cell gripper clamps 
the quartz cell out of the ultrasonic water bath filling port, and clamps it to the drying port of the temperature regulator for drying. The quartz cell gripper puts the cleaned and dried the quartz cell back into the quartz cell base.

\subsection{Constant Temperature Sterilization Storage Function}

As shown in figure 7 , the entire storage device includes two boxes, including a sterilizing $18^{\circ} \mathrm{C}$ constant temperature box and a sterilizing $4^{\circ} \mathrm{C}$ constant temperature box, which have functions of sterilization, temperature control and constant temperature storage. It can be ensured that the drug solution in the drug solution to be tested storage box, the carrier protein in the carrier protein storage box and the PBS buffer solution in the buffer storage box are properly stored at the storage temperature without changing the properties during the experiment. Therefore, the ultraviolet automatic sampling instrument can perform toxicity tests normally in any environment. It avoids the problem that the traditional manual sampling operation cannot complete the drug toxicity test experiment in a high or low-temperature environment.

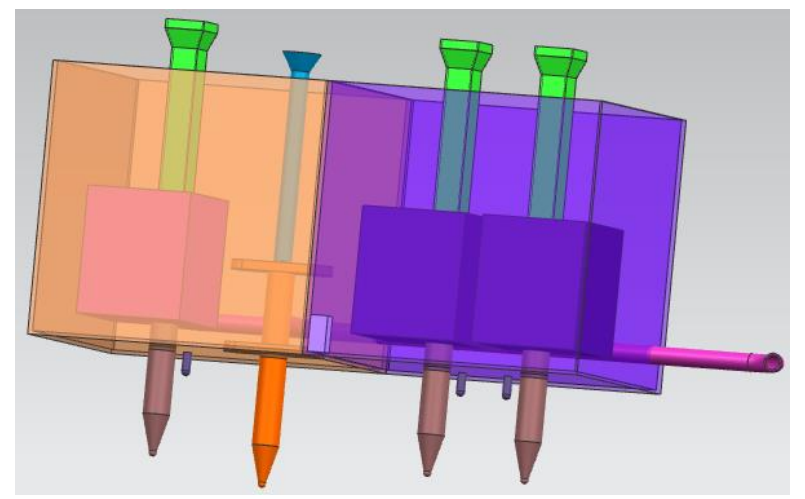

Figure 7: Constant temperature sterilization storage box parts diagram.

\subsection{Easy to Clean Structure-Function}

As shown in figure 8 , the bottom of the medicinal liquid in the medicinal liquid storage box to be tested, the protein in the protein storage box and the buffer liquid storage box are designed with a middle-high slope structure around the sides, which can be easily cleaned and does not leave the medicinal, protein or Buffer. Therefore, the UV sampler is easy to clean, and repeated use will not cause errors in the experiment.

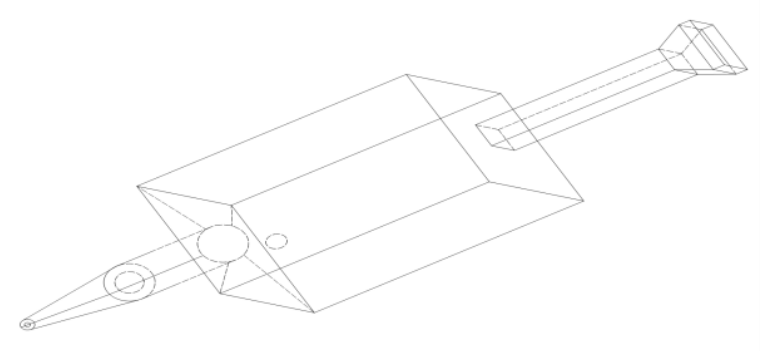

Figure 8: Box bottom structure diagram.

\subsection{Precise Sampling Function}

As shown in figure 9, position sensors are installed below the chemical storage box to be tested, the protein storage box and the buffer storage box. The quartz cell gripper can accurately clamp the quartz cell directly under the injection pipette head of the drug solution storage tank, carrier protein storage tank and buffer storage tank to be tested, and can accurately sample. This ensures that the sampler can accurately inject samples without not filling the outside of the quartz cell. 


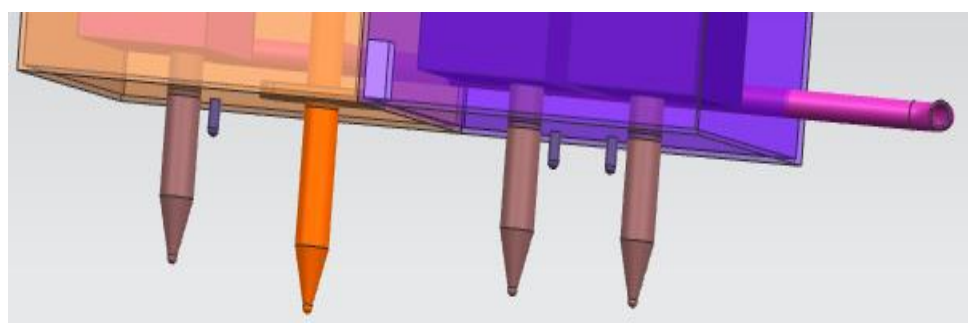

Figure 9: Position sensor parts diagram.

\subsection{Precise Heat Preservation and Temperature Control Function}

As shown in figure 10, the temperature regulator has temperature adjustment and drying functions, which can not only regulate the ambient temperature of the lower cavity of the device, but also dry the quartz cell. The Sterilization $18^{\circ} \mathrm{C}$ thermostat, the sterilization $4^{\circ} \mathrm{C}$ thermostat and the constant temperature water bath tube are installed with temperature sensors, which can accurately transmit temperature data to the temperature regulator in real-time, thereby monitoring the temperature Precise control. The insulation baffle is installed in the middle of the device. The obstacle sensor switch on the baffle will automatically open or close the heat preservation baffle through induction. The insulation baffle ensures that the quartz cell gripper, is clamped the quartz cell passed smoothly for sampling and return. Ensure that the ambient temperature of the lower cavity of the entire device is maintained at a specific temperature and the experimental results are not affected by temperature.

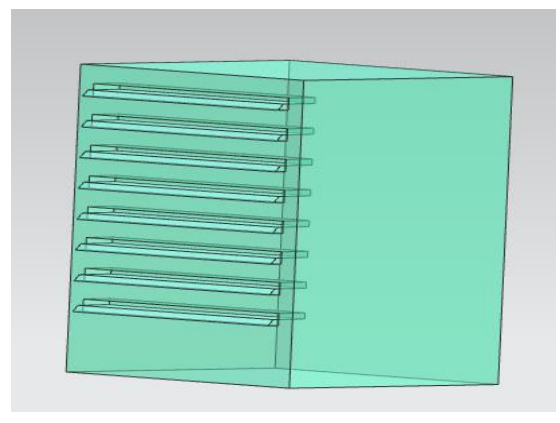

Figure 10: Temperature regulator parts diagram.

\subsection{Reciprocating Suction Mixing Function}

As shown in figure 11, the mixing gun directly below the drug solution to be tested storage box, the carrier protein storage box and the buffer storage box, the mixed liquid that can be sucked back and forth to ensure the PBS buffer, carrier protein the solution and the drug solution to be tested can be fully mixed to ensure the accuracy of the experiment.

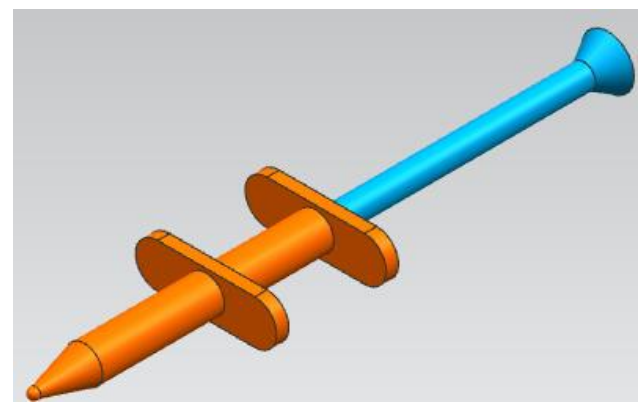

Figure 11: Mixing gun parts diagram.

\subsection{Artificial Intelligence Control}

As shown in figure 12, the ultraviolet automatic sampling instrument only needs simple installation, and all other steps are controlled by the artificial intelligence platform. Open the upper lid of the sample chamber of the "UV-1780 UV-Vis Spectrophotometer," and place the "Ultraviolet Automated 
Injector for Drug Toxicity Detection Based on the Information Angle of Binding to the Carrier Protein" on top of the sample chamber of the "UV-1780 UV-Vis Spectrophotometer". Combine the "UV-1780 UV-Vis Spectrophotometer" and the "Ultraviolet Automated Injector for Drug Toxicity Detection Based on the Information Angle of Binding to the Carrier Protein," and then connect them to the same computer through a USB jack. Import the "Platform for predicting drug toxicity based on the information angle of binding with carrier protein" software installation package into the computer, install and run.

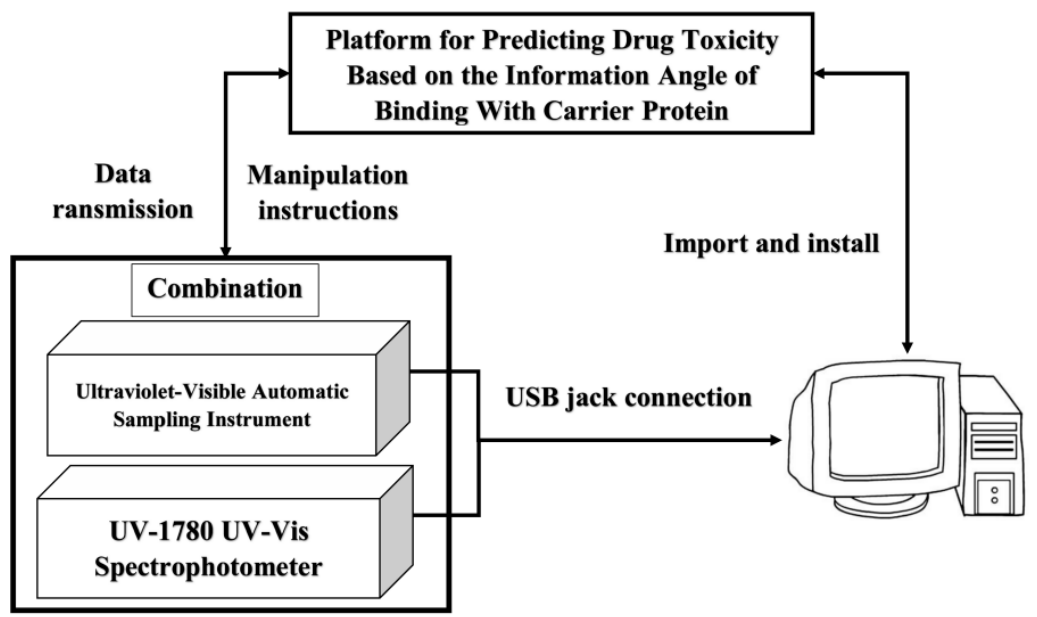

Figure 12: Schematic diagram of artificial intelligence control relationship.

\subsection{Artificial Intelligence Data Processing}

As shown in figure 13, after the experiment, the artificial intelligence platform will automatically acquire and process the experimental data, and obtain the spectroscopic parameter $r$ (combined distance) through Förster energy transfer theory. Then the platform substitutes the spectroscopy parameter $\mathrm{r}$ into the toxicity detection model of the platform, and then combines with other spectroscopy parameters to obtain the acute toxicity LD50 value and toxicity of the tested drug. At the same time, the fluorescence quenching mechanism can be preliminarily judged, and the changes in the secondary structure of the protein can be obtained and the modification opinions on the compound structure of the drug to be tested can be given.

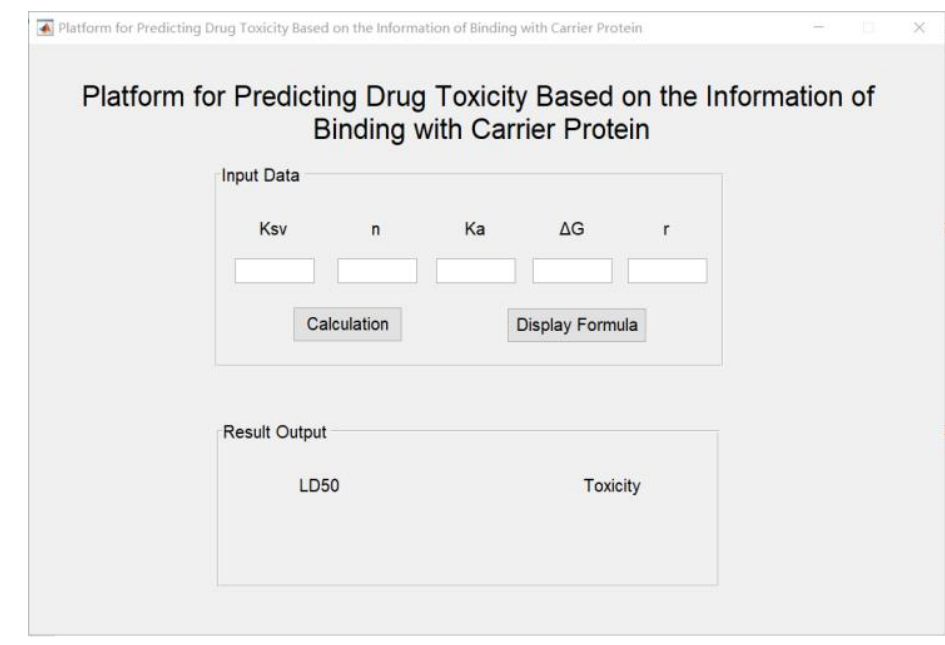

Figure 13: The operation interface of the drug toxicity detection platform based on the binding information of the carrier protein.

\section{Discussion}

This article specifically introduces the automatic sampler for UV spectroscopy toxicity detection, and it can be used as a tool to solve the low accuracy and long detection time because of its automatic 
sampling, sample mixing, automatic quartz cells cleaning and automatic control of temperature, sterility and other experimental background conditions. However, traditional UV spectrophotometers use manual sampling, the different skill levels of the operators, quartz cells cleaning, solution storage environment, temperature changes, reagent background interference and other factors. The measured spectral signals have a great Error range, poor accuracy and extended detection time. Among them, temperature has a greater limitation on the experimental results, which is the main cause of protein denaturation. In the design of fluorescence experiments, we often control the temperature of the drug's toxicity to human serum albumin within $37^{\circ} \mathrm{C}$ [4], and the temperature of the drug's toxicity to glutathione peroxidase is best controlled within $27^{\circ} \mathrm{C}[5]$. Otherwise, it is difficult to determine what is the dominant factor leading to changes in the carrier protein structure. Secondly, the test background ambient temperature has a great influence on the final sample result. Most UV spectrophotometers have difficulty controlling the environment temperature. For example, when the outside temperature is high or low, the temperature required for the test solution differs greatly from the ambient temperature. At this time, the accuracy of the toxicity detection of the tested liquid medicine will produce a large error.

In order to solve these problems, based on the "carrier protein binding information-toxicity relationship," the automatic sampler for UV spectroscopy toxicity detection is designed. The automatic sampling method is used to solve the test errors caused by the complex operation, solution storage environment, temperature changes and background interference, and meets the requirements of various drugs and the need to detect the toxicity of carrier protein. The ultraviolet automatic sampling instrument for toxicity detection has the characteristics of automation, high accuracy and fast detection speed, which realizes the rapid detection of a large number of drugs and lays the foundation for early toxicity prediction of drug development.

\section{Acknowledgments}

This work was supported by the Natural Science Foundation of Heilongjiang Province under Grant (grant number LH2019C055), the Project funded by China Postdoctoral Science Foundation under Grant (grant number 2019M651316), the Heilongjiang Provincial Postdoctoral Science Foundation under Grant (grant number LBH-Z18261), the Department of Education of Heilongjiang Province under Grant (grant numbers 2020-KYYWF-1031, 2020-KYYWF-1030, 2020-KYYWF-1029, 135409216), and Heilongjiang University Graduate Innovative Research Fund Project (grant number YJSCX2021-087HLJU).

\section{References}

[1] Zhou, F., Li, C., Zhu, H. and Li, Y. (2018) Simultaneous determination of trace metal ions in industrial wastewater based on uv-vis spectrometry. Optik - International Journal for Light and Electron Optics, doi: 10.1016/j.ijleo.2018.09.075.

[2] Passos, M. and Saraiva, M. (2019) Detection in uv-visible spectrophotometry: detectors, detection systems, and detection strategies. Measurement, 135, 896-904.

[3] Xing, Y., Wang, Z., Li. X., Hou, C., Chai, J., Li, X., Su, J., Gao, J. and Xu, H. (2021) A new method for predicting the acute toxicity of carbamate pesticides based on the perspective of binding information with carrier protein. Spectrochimica Acta Part A: Molecular and Biomolecular Spectroscopy, doi: 10.1016/j.saa.2021.120188.

[4] Wang, X., Xing, Y.. Su, J., Wang, C., Wang, Z., Yu, Y., Xu, H. and Ma, D. (2020) Synthesis of two new naphthalene-containing compounds and their bindings to human serum albumin. Journal of Biomolecular Structure and Dynamics, doi: 10.1080/07391102.2020.1764867.

[5] Xie, Y. and Hou, X. (2021) Molecular Assessment of the Toxic Mechanism of the Latest Neonicotinoid Dinotefuran with Glutathione Peroxidase 6 from Arabidopsis thaliana. Journal of Agricultural and Food Chemistry, 69 (2), 638-645. 